\title{
HÁ ESPAÇO PARA A ESTIMULAÇÃO ELÉTRICA NERVOSA TRANSCUTÂNEA (TENS) COM MEIO DE INCENTIVO A REGENERAÇÃO NERVOSA PERIFÉRICA? UM ENSAIO TEÓRICO
}

\author{
DM Cavalcante Programa de Pós-graduação em Medicina e Saúde, \\ Faculdade de Medicina, Universidade Federal da \\ Bahia, Salvador, BA, Brasil. Laboratório de \\ Eletroestimulação Funcional, Departamento de \\ Biomorfologia, Universidade Federal da Bahia, \\ Salvador, BA, Brasil. \\ EL Martins Programa de Pós-graduação em Medicina e Saúde, \\ Faculdade de Medicina, Universidade Federal da \\ Bahia, Salvador, BA, Brasil. Laboratório de \\ Eletroestimulação Funcional, Departamento de \\ Biomorfologia, Universidade Federal da Bahia, \\ Salvador, BA, Brasil. \\ AMB Martinez Laboratório de Neuroregeneração e Reparo, \\ Departamento de Histologia e Embriologia, \\ Universidade Federal do Rio de Janeiro, RJ, \\ Brasil. \\ MA Vannier-Santos \\ Laboratório de Biomorfologia Parasitária, Centro \\ de Pesquisas Gonçalo Moniz - FIOCRUZ, \\ Salvador, BA, Brasil. \\ Kátia Nunes Sá Pós-graduação, Pesquisa e Extensão, Escola \\ Bahiana de Medicina e Saúde Pública, Salvador, \\ BA, Brasil.

\begin{abstract}
AF Baptista Pós-graduação, Pesquisa e Extensão, Escola Bahiana de Medicina e Saúde Pública, Salvador, BA, Brasil. Laboratório de Biomorfologia Parasitária, Centro de Pesquisas Gonçalo Moniz FIOCRUZ, Salvador, BA, Brasil. Laboratório de Eletroestimulação Funcional, Departamento de Biomorfologia, Universidade Federal da Bahia, Salvador, BA, Brasil.
\end{abstract}

\begin{abstract}
Resumo
Introdução: $\mathrm{O}$ uso de campos eletromagnéticos com o intuito de se incentivar a regeneração nervosa periférica vem sendo estudado principalmente desde a década de 80. Mutios meios de se administrar campos elétricos foram utilizados, tais como eletrodos implantados, estimulação intra operatória e eletrodos de agulha, porém existem poucos dados acerca do uso da estimulação elétrica com eletrodos de superfície (transcutânea). Por outro lado esta forma é usada muitas vezes para o controle da dor, sendo que em algumas delas há lesão nervosa periférica e degeneração Waleriana associada. Objetivos: O objetivo deste trabalho é apresentar as formas de se usar campos elétricos clinicamente com o objetivo de se promover a regeneração nervosa periféricas e discutir, dentro deste contexo, o uso da estimulação elétrica nervosa transcutânea. Métodos: Foi realizada uma revisão narrativa da literatura, incluindo trabalhos pré clínicos e clínicos sobre o uso de campos elétricos para se promover regeneração nervosa periférica, que tenham sido publicados em periódicos indexados no PubMed e Scielo, nas línguas portuguesa, inglesa, esponhala e francesa, no período de 1980 até 2012 . Resultados e discussão: A grande maioria dos trabalhos encontrados são pré-clinicos, em camundongos e ratos. A estimulação peri-operatória ou com eletrodos implantados é a mais comum e somente um utilizou a estimulação elétrica nervosa transcutânea. Em geral os resultados apontam para um efeito positivo na regeneração nervosa
\end{abstract}


periférica, mas os parâmetros de estimulação parecem ser críticos para os resultados, especialmente a frequência da corrente, o período de início do tratamento e o número de sessões de estimulação.

\begin{abstract}
Introduction: The use of electromagnetic fields in order to promote peripheral nerve regeneration has been studied mainly from the $80 \mathrm{~s}$. Many ways of delivering electric fields were used, such as implanted electrodes, intraoperative stimulation and needle electrodes, but there are few data on the use of electrical stimulation with surface electrodes (transcutaneous). On the other hand this form is often used for pain control, and in some associated conditions, there is peripheral nerve injury and associated Waleriana degeneration. Objectives: The aim of this paper is to present ways of using electric fields clinically with the goal to promote peripheral nerve regeneration and discuss within this surrounding context, the use of transcutaneous electrical nerve stimulation. Methods: We performed a narrative review of the literature, including pre-clinical and clinical studies on the use of electric fields to promote peripheral nerve regeneration, which have been published in journals indexed in PubMed and SciELO, in Portuguese, English, French and spanish, from 1980 until 2012. Results and Discussion: The vast majority of studies found were preclinical in mice and rats. The perioperative stimulation with implanted electrodes is the most common form of stimulation, and only one used the transcutaneous electrical nerve stimulation. In general the results indicate a positive effect on peripheral nerve regeneration, but the stimulation parameters appear to be critical to the results, especially the frequency of the current, the period of initiation of treatment and the number of stimulation sessions.
\end{abstract}

Keywords: Peripheral nerve regeneration; Electrotherapy; Electric Fields; Transcutaneous electrical nerve stimulation.

\title{
INTRODUÇÃO
}

O Sistema Nervoso Periférico (SNP) pode ser lesado por traumas, distúrbios metabólicos, inflamatórios e muitos outros. Após uma lesão axonal ocorre extensa degeneração do segmento distal à lesão, conhecida por Degeneração Walleriana (DW). O coto proximal, que continua ligado ao corpo celular, pode se regenerar e crescer em direção ao órgão-alvo. ${ }^{(1)}$

As extremidades distais dos neurônios lesionados se dilatam pelo acúmulo de organelas citoplasmáticas e formam os "cones de crescimento". A partir desses cones são gerados brotos regenerativos, que crescem em direção ao órgão-alvo. O uso de correntes elétricas externas à lesão tem a finalidade de restabelecer o fluxo elétrico normal, aumentar a circulação local, aumentar o metabolismo celular e estimular a produção de fatores tróficos. ${ }^{(2)}$

O uso de eletrodos transcutâneos (de superfície) é uma opção não-invasiva e que pode ser utilizada por um período maior, especialmente quando associada à correntes bifásicas. Seu manejo é prático e simples, evitando a solução de continuidade e as reações provocadas por uma cirurgia de implante ou mesmo estimulação percutânea. Estudos prévios avaliaram a influência da Estimulação Elétrica Nervosa Transcutânea (TENS) na regeneração de tecidos como tendões, ${ }^{(3)}$ pele ${ }^{(4,5)}$ e osso, ${ }^{(6)}$ com efeitos variados. Resultados anteriores do nosso 
Cavalcante et al. Há espaço para a estimulação elétrica nervosa transcutânea (tens) com meio de incentivo a regeneração nervosa periférica? um ensaio teórico

laboratório mostraram que a TENS aplicada por um período de cinco semanas levou à inibição da regeneração nervosa periférica em camundongos. ${ }^{(7)}$

Os efeitos analgésicos da TENS estão relacionados à liberação de opióides endógenos $^{(8)}$ e a exposição crônica normalmente está associada com o desenvolvimento de tolerância farmacológica. Este fenômeno está associado à inibição de vários estágios da restauração do nervo periférico após lesão. ${ }^{(9)}$ Entretanto, a TENS pode aumentar o fluxo sanguíneo, ${ }^{(10)}$ que poderia incentivar a regeneração nervosa periférica se ela fosse aplicada de maneira a evitar o desenvolvimento de tolerância farmacológica

\section{LESÃO E REGENERAÇÃO NERVOSA PERIFÉRICA}

Seddon em 1943 e 1972 propôs uma classificação que descreve três estágios diferentes da lesão dos nervos. No primeiro grau de lesão há continuidade dos axônios, com bloqueio de condução local, e esta lesão corresponde às lesões por compressão, sendo a Neuropraxia o termo mais adequado para este tipo. ${ }^{(11)}$

O próximo grau de lesão, conhecido por Axoniotme, implica na perda da continuidade axonal no nível da lesão, porém com preservação dos tubos endoneurais. Corresponde ao avanço da lesão por compressão ou tração, quando são suficientes para interromper a continuidade axonal, resultando em DW do coto distal do nervo, causando paralisia motora, sensitiva e autonômica. E por fim, a lesão por Neurotmese que inclui a perda da continuidade de alguns ou todos os elementos do tronco nervoso, incluindo tubos endoneurais, perineuro e epineuro. ${ }^{(11)}$

Após uma lesão tecidual, um influxo de cálcio para dentro da célula provoca "correntes elétricas de lesão" através do aumento dos potenciais elétricos locais. ${ }^{(12)}$ As correntes de lesão modulam o processo de reparo e sua existência justifica o uso de correntes elétricas exógenas, com o fim de incentivar este processo e assim a recuperação tecidual precoce.

A regeneração axonal pode ser influenciada por pistas quimiotáxicas e elétricas, ${ }^{(13)} \mathrm{de}$ forma que se estas não forem adequadas ou a distância a ser percorrida pelos brotos axonais for muito grande, a regeneração pode não ser funcional. Isto acontece em lesões muito proximais, perto dos corpos celulares no SNP, como as lesões traumáticas no plexo braquial, lesões de raízes nervosas no forame de conjugação e outras. ${ }^{(14)}$ Também em situações onde o metabolismo ou o fornecimento de nutrientes está acometido, como na Diabete Melito (DM) 
Cavalcante et al. Há espaço para a estimulação elétrica nervosa transcutânea (tens) com meio de incentivo a regeneração nervosa periférica? um ensaio teórico

há a dificuldade de se desenvolver uma regeneração funcional. ${ }^{(15)}$ Nestas situações o incentivo à regeneração se torna uma necessidade, para que o organismo acometido volte a desempenhar as funções normais, perdidas após a lesão.

Para incentivar a regeneração no nervo periférico, pode-se recorrer a diversos recursos, com o uso de fatores neurotróficos, células-tronco, ${ }^{(16)}$ aplicação de ultra-som, ${ }^{(17)}$ LASER de baixa intensidade, ${ }^{(18)}$ exercícios físicos ${ }^{(19)}$ e outros. Dentro deste rol de opções o uso de campos eletromagnéticos constitui uma das possibilidades, que se justifica pelo fato de que após uma lesão tecidual em um organismo biológico se formam correntes elétricas endógenas, que estão intimamente relacionadas com o processo de recuperação. Estas correntes, conhecidas como Correntes de Lesão (CL), são formadas através de gradientes elétricos entre a área acometida e as regiões vizinhas e permanecem ativas durante todo o processo regenerativo. ${ }^{(20)}$

\section{CAMPOS ELÉTRICOS E REGENERAÇÃO NERVOSA PERIFÉRICA}

A manipulação da regeneração nervosa periférica no sentido de facilitar a reinervação inclui a aplicação de campos elétricos constantes ou com frequências variadas. A efetividade das condutas que visam aumentar a taxa e a velocidade de regeneração tem sido demonstrada em vários trabalhos, eminentemente com animais de experimentação.

São utilizadas normalmente correntes elétricas monofásicas (pulsadas ou não) ou bifásicas, administradas através de eletrodos implantados no próprio nervo, estimulação intraoperatória ou percutânea, usando agulhas semelhantes às de acupuntura ligadas a um gerador de corrente. As correntes monofásicas possuem a vantagem da unidirecionalidade, que pode gerar efeitos eletroforéticos nas proteínas de membrana e, desta forma, orientar o crescimento do neurito em direção ao cátodo. ${ }^{(12)}$ Entretanto, se forem usadas em altas intensidades ou longos períodos podem causar efeitos lesivos devido à própria eletroforese. ${ }^{(20)}$ Apesar das correntes bifásicas não oferecerem o risco dos efeitos decorrentes da eletrólise, elas são menos utilizadas, pois não possuem poder de gerar o direcionamento do neurito para o órgão-alvo.

As formas de aplicação das correntes elétricas com objetivo de estimular a regeneração nervosa incluem diferentes tipos de eletrodos, com algumas formas predominando sobre outras. Em geral, quanto mais perto o eletrodo estiver do nervo, a 
Cavalcante et al. Há espaço para a estimulação elétrica nervosa transcutânea (tens) com meio de incentivo a regeneração nervosa periférica? um ensaio teórico

corrente tende a ser menor em amplitude, pois não há necessidade de vencer a impedância exercida pelos tecidos circundantes do nervo.

\section{Eletrodos implantados}

A estimulação com eletrodos implantados e diferentes tipos de correntes elétricas tem sido a forma mais utilizada desde a década de 80 em modelos experimentais usando lesões nos nervos sóleo ${ }^{(21)}$ e isquiático. ${ }^{(22)}$ Todos esses trabalhos apresentaram resultados positivos, com melhora na função e/ou parâmetros morfológicos.

Dois estudos, realizados pelo mesmo grupo, utilizando como modelo a lesão do nervo fibular comum ${ }^{(24)}$ e do isquiático ${ }^{(23)}$ encontraram resultados negativos, com menor número de fibras em regeneração, formação de neuromas e taxa mais baixa de mielinização nos grupos estimulados. Entretanto, em seus experimentos um dos eletrodos foi colocado dentro do pequeno tubo de silicone que unia os dois cotos seccionados, levando à interação da corrente elétrica monofásica com o material do tubo, formação de efeitos deletérios pela falta de alternância da corrente e consequentemente destruição de parte do tubo, com impedimento físico à passagem dos neuritos.

As correntes elétricas utilizadas em todos estes estudos foram monofásicas e as amplitudes de corrente sempre muito baixas, abaixo do limiar sensitivo (na faixa dos $\mu \mathrm{A}$ ), para que não houvesse destruição tecidual. A estimulação era feita geralmente de forma contínua, por períodos de algumas semanas, antes que os animais fossem sacrificados e as análises realizadas.

\section{Eletroestimulação percutânea}

Os eletrodos percutâneos normalmente são agulhas de acupuntura introduzidas na pele e ligadas a um gerador de corrente elétrica. O uso de correntes elétricas através de eletrodos percutâneos tem sido pesquisado em modelos experimentais e talvez seja dos métodos estudados até o momento, o mais simples de se aplicar na prática clínica. Este tipo de estimulação foi estudado em modelos de lesão do nervo isquiático, ${ }^{(25)} \mathrm{com}$ os melhores resultados sendo atingidos quando o cátodo foi colocado distal e o ânodo proximal à lesão. Nestes experimentos foram sempre utilizadas correntes monofásicas, com amplitudes também 
Cavalcante et al. Há espaço para a estimulação elétrica nervosa transcutânea (tens) com meio de incentivo a regeneração nervosa periférica? um ensaio teórico

abaixo do limiar sensitivo, entretanto tendo chegado à intensidade de um miliampére, bem maior do que aquela utilizada nas técnicas com eletrodos implantados.

\section{Eletroestimulação intra-operatória}

Nestes modelos, o nervo é estimulado logo após a lesão, por períodos variáveis. Os nervos mais utilizados nos estudos são o isquiático ${ }^{(26)}$ e o femoral. ${ }^{(27)}$ Os primeiros estudos utilizaram correntes monofásicas e os mais recentes, correntes alternadas com frequências de pulso de $20 \mathrm{~Hz} .{ }^{(28)}$ Com esse último modelo, os autores encontraram uma reinervação mais precisa e mais rápida de órgãos-alvo, aumento na expressão do BDNF, TrKB, das proteínas Ta1-tubulina e GAP-43 e de genes associados à regeneração, além de redução na expressão de neurofilamentos (fenômeno também associado à regeneração). Este modelo foi o único até o momento a ser extrapolado para seres humanos. Gordon et al. ${ }^{(14)}$ avaliaram o mesmo protocolo de estimulação elétrica em pacientes que possuíam denervação dos músculos da região tenar consequente a Síndrome do Túnel do Carpo e encontraram que o tratamento acelerou a regeneração axonal sem afetar a função manual. Foecking et al. ${ }^{(12)}$ realizaram um estudo recente com estimulação intra-operatória e demonstraram que o fator mais importante para a regeneração nervosa periférica é o início precoce da estimulação e não o quanto ela é repetida através dos dias de regeneração. ${ }^{(12)}$ A eletroestimulação intra-operatória se diferencia das outras, além da forma de estimulação, pelo tempo breve que é empregado, em torno de uma a duas horas de estimulação após a lesão.

\section{Eletrodos de superfície (eletroestimulação transcutânea)}

O uso de eletrodos de superfície é uma opção não-invasiva e que pode ser utilizada por um período maior, especialmente quando associados à corrente bifásica. Seu manejo é prático e simples, evitando a solução de continuidade e as reações provocadas por uma cirurgia de implante ou mesmo estimulação percutânea.

Estudos prévios avaliaram a influência da TENS na regeneração de tecidos como tendões ${ }^{(3)}$ pele $^{(5)}$ e osso, ${ }^{(6)}$ com resultados variados. Esta modalidade tem sido associada com efeitos como o aumento no fluxo sanguíneo, ${ }^{(10)}$ mas é necessário usar correntes elétricas com amplitudes acima de $1 \mathrm{~mA}$, que podem ser relacionadas à diminuição nas concentrações de adenosinatrifosfato (ATP) ${ }^{(31)}$ e consequentemente inibição na regeneração. 
Cavalcante et al. Há espaço para a estimulação elétrica nervosa transcutânea (tens) com meio de incentivo a regeneração nervosa periférica? um ensaio teórico

Os efeitos dos campos elétricos na circulação de sangue promovem aumento do brotamento axonal e regeneração nervosa. ${ }^{(32)}$ Correntes elétricas de baixa frequência podem ativar seletivamente fibras sensoriais $\mathrm{C}$ e aumentar a expressão de neuropeptídeos tais como Substância P, que então geram vasodilatação. ${ }^{(33)}$ A TENS de baixa frequência $(2$ a $10 \mathrm{~Hz})$ é usualmente associada com contração muscular rítmica, que também pode ter efeitos circulatórios. ${ }^{(34)}$ Entretanto, evidências recentes indicam que a TENS de alta frequência, que é usada sem produção de contração muscular, também tem efeito de aumento na circulação. De Vries e colaboradores ${ }^{(10)}$ demonstraram um aumento na circulação coronária consequente a TENS de alta frequência. Por outro lado, deve ser considerado que a exposição a campos eletromagnéticos e consequente aumento na irrigação associado ou não a angiogênese não é sempre positiva, pois pode estar relacionada ao aumento na concentração local de espécies reativas de oxigênio ${ }^{(35)}$ e lesão no DNA. ${ }^{(36)}$

Os efeitos mais estudados da TENS são em relação ao controle da dor. A analgesia provocada pela TENS de alta frequência está ligada à ativação de receptores $\delta$-opióides, enquanto que a TENS de baixa frequência ativa receptores $\mu$-opióides. ${ }^{(8)}$ Sinatra e Ford $^{(9)}$ demonstraram que o uso crônico de morfina por 14 dias levou a um atraso na regeneração nervosa periférica, expressa por uma menor quantidade de perfis axonais, diminuição na remoção de restos de mielina, hipertrofia e proliferação de células de Schwann. Zeng et al. ${ }^{(37)}$ também demonstraram que a exposição à morfina, agindo via receptores $\mu$-opióides, aumentou a regeneração de fibras não mielinizadas, mas inibiu a de fibras mielinizadas após lesão por esmagamento do nervo isquiático. Isto está de acordo com resultados prévios de nosso laboratório, ${ }^{(7)}$ nos quais as fibras em regeneração não mielinizadas não foram afetadas pela TENS, mas as mielinizadas de grosso calibre estiveram presentes em menor quantidade e apresentando menos conteúdo mielínico.

A diferença fundamental entre os estudos que mostraram efeitos estimulantes ou inibitórios na regeneração do SNP com a ativação de receptores opióides foi o tempo prolongado de utilização, que pode ter levado ao desenvolvimento de tolerância farmacológica. Chandran e Sluka ${ }^{(38)}$ demonstraram que a administração repetida de TENS de alta e baixa frequência, 20 minutos por dia, levou a tolerância a opióides no quarto dia de estimulação. Mao et al. ${ }^{(39)}$ demonstraram que ela é mediada pela via NMDA-caspase e leva a apoptose de células neuronais na medula espinhal. Portanto, o uso prolongado da TENS pode levar ao desenvolvimento de tolerância a opióides e consequências neurotóxicas para as células envolvidas na regeneração. Entretanto um uso breve, que não levasse à tolerância 
Cavalcante et al. Há espaço para a estimulação elétrica nervosa transcutânea (tens) com meio de incentivo a regeneração nervosa periférica? um ensaio teórico

opióide, poderia ser um importante fator de incentivo à regeneração. Estudo recente tem demonstrado que o período breve de aplicação da estimulação elétrica, realmente é determinante para a regeneração nervosa periférica, no entanto neste estudo a estimulação foi intraoperatória. ${ }^{(30)}$

Diante deste ensaio teórico, nós tentamos demonstrar os diferentes desfechos da aplicação da TENS para incentivar a regeneração nervosa periférica, visando estimular a realização de novos estudos experimentais e principalmente ensaios clínicos com este tema. Acreditamos que o momento ideal, bem como o número de sessões da TENS são pontos chaves para a utilização deste recurso como terapia para incentivar a regeneração nervosa periférica.

\section{REFERÊNCIAS}

1. Stoll G, Müller HW. Nerve injury, axonal degeneration and neural regeneration: Basic. Brain Pathol. 1999; 9(2): 313-325.

2. Panagopoulos DJ, Karabarbounis A, Margaritis LH. Mechanism for action of electromagnetic fields on cells. Biochem. Biophys. Res. Commun. 2002; 298: 95-102.

3. Burssens P, Forsyth R, Steyaert A, Van Ovost E, Praet M, Verdonk R. Influence of burst TENS stimulation on the healing of Achilles tendon in man. Acta Orthop Belg. 2003; 69(6): 528-532.

4. Khalil Z, Merhi M. Effects of aging on neurogenic vasodilator responses evoked by transcutaneous electrical nerve stimulation: relevance to wound healing. J. Gerontol. 2000; 55(6): 257-263.

5. Liebano RC, Ferreira LM, Neto MS. Experimental model for transcutaneous electrical nerve stimulation on ischemic random skin flap in rats. Acta cir. bras. 2003; 18: 54-59.

6. Kahn J. Transcutaneous electrical nerve stimulation for nonunited fractures; a clinical report. Physical Therapy. 1982; 62(6): 840-844.

7. Baptista AF, Gomes JRS, Oliveira JT, Santos SMG, Vannier-Santos MA, Martinez AMB. High and low frequency transcutaneous electrical nerve stimulation delay sciatic nerve regeneration after crush lesion in the mouse. J. Peripher. Nerv. Syst. 2008; 13: 71-80.

8. Sluka KA, Walsh D. Transcutaneous electrical nerve stimulation: Basic science mechanisms and clinical effectiveness. J. Pain. 2003; 4(3): 109-121.

9. Sinatra RS, Ford DH. The effects of acute and chronic morphine treatment on the process of facial nerve regeneration. Brain Res. 1979; 175(2): 315-325. 
Cavalcante et al. Há espaço para a estimulação elétrica nervosa transcutânea (tens) com meio de incentivo a regeneração nervosa periférica? um ensaio teórico

10. De Vries J, Anthonio RL, Dejongste MJ, Jessurun GA, Tan ES, De Smet BJ et al. The effect of electrical neurostimulation on collateral perfusion during acute coronary occlusion. BMC Cardiovasc Disord. 2007; 27: 7-18.

11. Siqueira R. Lesões nervosas periféricas: uma revisão. Rev Neurocienc. 2007; 3: 219-225.

12. McCaig CD, Rajnicek AM, Song B, Zhao M. Has electric growth cone guidance found its potential? Trends Neurosci. 2002; 25(7): 354-359.

13. Song B, Zhao M, Forrester J, McCaig C. Nerve regeneration and wound healing are stimulated and directed by an endogenous electric field in vivo. J. Cell. Sci. 2004; 117: 46811690 .

14. Gordon T, Sulaiman O, Boyd JG. Experimental strategies to promote functional recovery after peripheral nerve injuries. J. Peripher. Nerv. Syst. 2003; 8: 236-250.

15. Kennedy JM, Zochodne DW. Impaired peripheral nerve regeneration in diabetes mellitus. J. Peripher. Nerv. Syst. 2005; 10(2): 144-157.

16. Lopes FRP, Campos LCM, Corrêa Júnior JD, Balduino A, Lora S, Langone, F et al. Bone marrow stromal cells and resorbable collagen guidance tubes enhance sciatic nerve regeneration in mice. Exp Neurol. 2006; 198: 457-468.

17. Crisci AR, Ferreira AL. Low-intensity pulsed ultrasound accelerates the regeneration of the sciatic nerve after neurotomy in rats. Ultrasound Med Biol. 2002; 28(10): 1335-1341.

18. Bae CS, Lim SC, Kim KY, Song CH, Pak S, Kim M et al. Effect of Ga-As laser on the regeneration of injured sciatic nerves in the rat. In vivo, 2004; 18(4): 489-495.

19. Seo TB, Han IS, Yoon JH, Hong KE, Yoon SJ, Namgung, U. Involvement of Cdc2 in axonal regeneration enhaced by exercise trainning in rats. Med Sci Sports Exerc. 2006; 38(7): $1267-1276$.

20. Low J, Reed A. Eletroterapia Explicada. São Paulo: Manole; 2001. p. 472.

21. Nix WA, Hopf HC. Electrical stimulation of regenerating nerve and its effect on motor recovery. Brain Res. 1983; 272: 21-25.

22. Mendonça AC, Barbieri CH, Mazzer N. Directly applied low intensity direct current enhances peripheral nerve regeneration in rats. J Neurosci Methods. 2003; 129: 183-190.

23. Hanson SM, McGinnis ME. Regeneration of rat sciatic nerves in silicone tubes: characterization of the response to low intensity d.c. stimulation. Neuroscience. 1994; 58(2): 411-421.

24. McGinnis ME, Murphy DJ. The lack of an effect of applied d.c. electric fields on peripheral nerve regeneration $n$ the guinea pig. Neuroscience. 1992; 51(1): 231-244. 
Cavalcante et al. Há espaço para a estimulação elétrica nervosa transcutânea (tens) com meio de incentivo a regeneração nervosa periférica? um ensaio teórico

25. Inoue M, Hojo T, Yano T, Katsumi Y. The effects of electroacupuncture on peripheral nerve regeneration in rats. Acupunct Med. 2003; 21(1-2): 9-17.

26. Scott JJA. Recovery of denervated muscle receptors following treatments to accelerate nerve regeneration. Brain Res. 1991; 563: 195-202.

27. Al-Majed AA, Tam SL, Gordon T. Electrical stimulation accelerates and enhances expression of regeneration-associated genes in regenerating rat femoral motoneurons. Cell. Mol. Neurobiol. 2004; 24(3): 379-402.

28. Brushart TM, Hoffman PN, Royall RM, Murinson BB, Witzel C, Gordon T. Electrical stimulation promotes motoneuron regeneration without increasing its speed or conditioning the neuron. Journal of Neuroscience. 2002; 22(15): 6631-6638.

29. Gordon T, Amirjani N, Edwards DC, Chan KM. Brief post-surgical electrical stimulation accelerates axon regeneration and muscle reinnervation without affecting the functional measures in carpal tunnel syndrome patients. Exp. Neurol. 2010; 223: 192-202.

30. Foecking E, Fargo K, Coughlin L, Kim J, Marzo S, Jones K. Single session of brief electrical stimulation immediately following crush injury enhances functional recovery of rat facial nerve. JRRD, 2012; 49 (3): 451-458.

31. Cheng N, Van Hoof H, Bockx E, Hoogmartens MJ, Mulier JC, De Dijcker FJ et al. The effects of electric currents on ATP generation, protein synthesis, and membrane transport of rat skin. Clinicial Orthopaedics and Related Research. 1982; 171: 264-272.

32. McCaig CD, Rajnicek AM, Song B, Zhao M. Controlling cell behavior electrically: Current views and future potential. Physiol. Rev. 2005; 85: 943-978.

33. Kashiba H, Ueda Y. Acupuncture to the skin induces release of Substance P and calcitonin gene-related peptide from peripheral nerve terminals of primary sensory neurons in the rat. Am J Chin Med. 1991; 19(3-4): 189-197.

34. Dobsák P, Nováková M, Siegelová J, Fiser B, Vitovec J, Nagasaka M et al. Lowfrequency electrical stimulation increases muscle strength and improves blood supply in patients with chronic heart failure. Circ. J. 2006; 70(1): 75-82.

35. Sauer H, Bekhite MM, Hescheler J, Wartenberg M. Redox control of angiogenic factors and CD31-positive vessel-like structures in mouse embryonic stem cells after direct current electric field stimulation. Exp Cell Res. 2005; 304(2): 380-390.

36. Delimaris J, Tsilimigaki S, Messini-Nicolaki N, Ziros E, Piperakis SM. Effects of pulsed electric fields on DNA of human lymphocytes. Cell Biol. Toxicol. 2006; 22(6): 409-415. 
Cavalcante et al. Há espaço para a estimulação elétrica nervosa transcutânea (tens) com meio de incentivo a regeneração nervosa periférica? um ensaio teórico

\section{Zeng et al.}

38. Chandran P, Sluka KA. Development of opioid tolerance with repeated transcutaneous electrical nerve stimulation administration. J. Pain, 2003; 102(1-2): 195-201.

39. Mao J, Sung B, Ji RR, Lim G. Neuronal apoptosis associated with morphine tolerance: Evidence for an opioid-induced neurotoxic mechanism. Journal of Neuroscience. 2002; 22(17): 7650-7661. 